\title{
On maximizing measures of homeomorphisms on compact manifolds
}

\author{
by
}

\author{
Fábio Armando Tal and Salvador Addas-Zanata (São Paulo)
}

\begin{abstract}
We prove that given a compact $n$-dimensional connected Riemannian manifold $X$ and a continuous function $g: X \rightarrow \mathbb{R}$, there exists a dense subset of the space of homeomorphisms of $X$ such that for all $T$ in this subset, the integral $\int_{X} g d \mu$, considered as a function on the space of all $T$-invariant Borel probability measures $\mu$, attains its maximum on a measure supported on a periodic orbit.
\end{abstract}

1. Introduction. Optimization problems are of great interest in mathematics. To maximize certain objects (functions, functionals, etc.) under given constraints is in general a difficult problem; such problems have given rise to several areas in mathematics like variational calculus, convex optimization or Lagrangian mechanics. In Lagrangian mechanics, for instance, solutions satisfy a certain variational principle: they are stationary points of the action integral. In particular, local minima or maxima for this functional are solutions.

Moreover, following Mather, orbits with prescribed properties can be obtained by considering minimizing measures (those that minimize the integral of the Lagrangian) in certain homology classes, and the orbits are obtained as typical points for these (ergodic) measures (see for instance [7]).

One can also consider a discrete version of this problem, namely given a topological space $X$, a continuous surjective mapping $T: X \rightarrow X$ and a continuous function $g: X \rightarrow \mathbb{R}$, look at $\sup _{\mu \in M_{\text {inv }}(T)} \int_{X} g d \mu$, where $M_{\text {inv }}(T)=\{T$-invariant Borel probability measures $\}$, which is a non-empty set by Krylov-Bogolyubov's theorem. This supremum is realized by an ergodic measure, and an interesting question, albeit still poorly understood, is to describe the interplay between the mapping $T$, the target function $g$

2000 Mathematics Subject Classification: 37A05, 37B99, 46A55.

Key words and phrases: periodic orbits, cocycles, Minkowski function, sources.

Addas-Zanata and Tal are partially supported by CNPq, grants: 301485/03-8 and 304360/05-8 respectively. 
and the ergodic measure. The work done so far concerns the question: keeping the mapping $T$ fixed, how does the maximizing measure change as the function $g$ varies? In particular, there is a considerable amount of work on this problem when $T$ is a hyperbolic or expanding mapping (for instance for the doubling mapping on the circle); see [3]. The main conjecture here is that, for a generic Lipschitz function $g$, the maximizing measure is supported on a periodic orbit (see [5]). Another line of research is to describe for which ergodic measures $\mu$ there exists a target function $g_{\mu}$ such that $\mu$ is the unique maximizing measure (see [4]).

The problem we want to study here goes in the opposite direction: we fix $g$ and vary $T$ and try to understand what happens to the maximizing measure, that is, we try to understand how the maximizing measure transforms when the dynamics is changed.

The main difference between these two discrete settings and Lagrangian mechanics is that in the Lagrangian case, the orbits and the Lagrangian are related by the Euler-Lagrange equation, while $T$ and $g$ can be perturbed independently.

In the following we will give a precise explanation of the problem studied in this paper, and the main result will be formulated.

Let $X$ be a compact $n$-dimensional connected Riemannian manifold and let $T: X \rightarrow X$ be a homeomorphism. Given a continuous function $g$ : $X \rightarrow \mathbb{R}$, one can study the following problem: As $T$ is a homeomorphism of a compact metric space, Krylov-Bogolyubov's theorem asserts that the set $M_{\mathrm{inv}}(T)$ of $T$-invariant Borel probability measures is non-empty. So, we consider the number

$$
Q(g)=\sup _{\mu \in M_{\mathrm{inv}}(T)} \int_{X} g d \mu .
$$

Since $M_{\text {inv }}(T)$ is convex and compact in the weak* topology, there exists at least one $\nu \in M_{\text {inv }}(T)$ (called a $g$-maximizing measure) such that

$$
Q(g)=\int_{X} g d \nu
$$

As the ergodic measures are exactly the extreme points of $M_{\text {inv }}(T)$, there must be at least one ergodic $g$-maximizing measure, and so we can assume that $\nu$ is ergodic.

In the space of homeomorphisms of $X$, denoted by $\operatorname{Hom}(X)$, we define a distance in the following natural way: given $T, F \in \operatorname{Hom}(X)$,

$$
d_{\text {Hom }}(T, F)=\max _{x \in X} d(T(x), F(x)), \quad \text { where } d \text { is a distance in } X .
$$

Note that $d_{\text {Hom }}$ is topologically equivalent to

$$
D_{\text {Hom }}(T, F)=\max \left\{\max _{x \in X} d(T(x), F(x)), \max _{x \in X} d\left(T^{-1}(x), F^{-1}(x)\right)\right\},
$$

a distance which makes $\operatorname{Hom}(X)$ a complete metric space. 
Now we are ready to state our theorem:

Theorem 1. For a fixed continuous function $g: X \rightarrow \mathbb{R}$, there exists a dense subset of $\operatorname{Hom}(X)$ such that for all $T$ in this subset, there is a $g$-maximizing $T$-invariant measure supported on a periodic orbit.

This result suggests some other natural questions, for instance:

1) What else can be said about the set of homeomorphisms that have a $g$-maximizing $T$-invariant measure supported on a periodic orbit? A simple argument shows that this set is not open, but is it residual or does it even have interior points?

2) As there is a $\mathcal{C}^{1}$-closing lemma, is it possible to prove a $\mathcal{C}^{1}$ version of the above theorem? This seems to be a very hard question, our methods do not generalize to the $\mathcal{C}^{1}$ world. The first part of the proof, which consists of closing a recurrent orbit in order to create a periodic one with larger average, seems to be very hard to generalize to the $\mathcal{C}^{1}$ world, even if we consider Mañé's ergodic version of the closing lemma, [6]. And even worse, the second part of the proof is a $\mathcal{C}^{0}$ only argument.

3) What happens when $T$ is just surjective, but not a homeomorphism? We solved this problem in the case of the circle, but in higher dimensions many difficulties appear.

This paper is organized as follows. In the next section we present some results about cocycles and in the last section we give the proof of the main theorem.

2. Basic tools. Here we present some results and ideas from the theory of cocycles of ergodic transformation groups. Fundamental references in this subject are the book of Schmidt [8] and the paper of Atkinson [2]. Another source for these results, but in a somewhat different formulation, is the book by Aaronson [1].

We state the results not in full generality, but only in the generality needed for our application.

Given $T \in \operatorname{Hom}(X)$, a $T$-invariant ergodic probability measure $\mu$ and a continuous function $\varphi: X \rightarrow \mathbb{R}$, we define the cocycle for $T$ given by $\varphi$ as the function $a: \mathbb{Z} \times X \rightarrow \mathbb{R}$ given by

$$
a(n, x)= \begin{cases}\sum_{i=0}^{n-1} \varphi \circ T^{i}(x) & \text { for } n>0, \\ 0 & \text { for } n=0, \\ -a\left(-n, T^{n}(x)\right) & \text { for } n<0 .\end{cases}
$$

The skew-product extension of $T$, determined by $\varphi$, is given by the following mapping $V: X \times \mathbb{R} \rightarrow X \times \mathbb{R}$ : 


$$
V(x, t)=(T(x), t+\varphi(x))
$$

So, the powers of $V$ can be expressed as

$$
V^{n}(x, t)=\left(T^{n}(x), t+a(n, x)\right) .
$$

An important definition is the following: The cocycle $a$ is recurrent if for every $B \in \sigma_{\mathcal{B}}(X)=$ Borel $\sigma$-algebra of $X$, with $\mu(B)>0$ and every $\varepsilon>0$, there is an $n \neq 0$ such that

$$
\mu\left(B \cap T^{-n}(B) \cap\{x:|a(n, x)|<\varepsilon\}\right)>0 .
$$

The following is a result from [2]:

Theorem 2. Suppose $\left(X, \sigma_{\mathcal{B}}(X), \mu\right)$ is a non-atomic probability space, $T \in \operatorname{Hom}(X)$ is ergodic with respect to $\mu$ and $\varphi: X \rightarrow \mathbb{R}$ is a continuous function such that $\int_{X} \varphi d \mu=0$. Then the cocycle a for $T$ determined by $\varphi$ is recurrent.

It is easy to see that the skew-product $V$ (see (2)) leaves the product measure $\mu \times \lambda$ invariant, where $\lambda$ is the Lebesgue measure in $\mathbb{R}$. The problem here is that the space $X \times \mathbb{R}$ is not compact, so we need to work a little more in order to get some kind of recurrence (to be more precise, topological recurrence) for $V$. An important definition for this purpose is the following (see Schmidt [8, Chapter 1]):

The skew-product $V$ is conservative if for every $A \in \sigma_{\mathcal{B}}(X \times \mathbb{R})$ with $\mu \times \lambda(A)>0$ and for $\mu \times \lambda$-a.e. $(x, t) \in A$, the set

$$
\left[\bigcup_{n \in \mathbb{Z}} V^{n}(x, t)\right] \cap A
$$

is infinite. Finally, we present a theorem relating the concepts of recurrence of the cocycle and conservativity of the skew-product (see [8, Chapter 5]):

TheOrem 3. Suppose $\left(X, \sigma_{\mathcal{B}}(X), \mu\right)$ is a non-atomic probability space, the homeomorphism $T \in \operatorname{Hom}(X)$ is ergodic with respect to $\mu$ and the cocycle $a$ is recurrent. Then the skew-product $V$ given by (2) is conservative.

So, Theorems 2 and 3 imply that for any continuous function $\varphi: X \rightarrow \mathbb{R}$ such that $\int_{X} \varphi d \mu=0$, the cocycle $a(n, x)$ is recurrent and the skew-product $V(x, t)$ is conservative. A simple consequence of the conservativity of a skewproduct $V$ as in (2) is the following:

LEMMA 1. If a skew-product $V$ as in (2) is conservative, then given any $B \in \sigma_{\mathcal{B}}(X)$ with $\mu(B)>0$ and any $\delta>0$, for $\mu$-a.e. $x \in B$ we have $T^{n}(x) \in B$ and $|a(n, x)|<\delta$, for infinitely many $n \in \mathbb{Z}$.

Proof. Immediate from the definitions. 
3. Proof of the main theorem. By hypothesis, $X$ is a connected compact Riemannian manifold, $T \in \operatorname{Hom}(X), g: X \rightarrow \mathbb{R}$ is a continuous function. Suppose that $T$ does not have periodic $g$-maximizing measures and let $\mu \in M_{\mathrm{inv}}(T)$ be an ergodic $g$-maximizing measure, which by the previous assumption is clearly non-atomic.

The following is a fundamental set for our construction:

$$
A=\left\{x \in \operatorname{supp}(\mu): x \text { is } T \text {-recurrent and } \frac{1}{N} \sum_{i=0}^{N-1} g \circ T^{i}(x) \stackrel{N \rightarrow \infty}{\longrightarrow} Q(g)\right\}
$$

By saying that $x$ is T-recurrent, we mean the usual: there exists a sequence $n_{i} \rightarrow \infty$ as $i \rightarrow \infty$ such that $d\left(T^{n_{i}}(x), x\right) \rightarrow 0$ as $i \rightarrow \infty$. Clearly, $\mu(A)=1$ and $T(A)=A$. Without loss of generality, by considering $g_{0}(x):=g(x)-$ $Q(g)$ we have

$$
\int_{X} g_{0} d \mu=0 .
$$

So, Theorems 2 and 3 imply that the cocycle

$$
a(n, x)= \begin{cases}\sum_{i=0}^{n-1} g_{0} \circ T^{i}(x) & \text { for } n>0 \\ 0 & \text { for } n=0 \\ -a\left(-n, T^{n}(x)\right) & \text { for } n<0\end{cases}
$$

is recurrent and the skew-product

$$
V(x, t)=\left(T(x), t+g_{0}(x)\right)
$$

is conservative.

Lemma 2. For a given $\varepsilon>0$ and for all $x \in A$, there exists $0<\varepsilon^{*}=$ $\varepsilon^{*}(x)<\varepsilon / 10$ such that $T\left(B_{\varepsilon^{*}}(x)\right) \cap B_{\varepsilon^{*}}(x)=\emptyset$ (where $B_{\delta}(x)=\{y \in X$ : $d(y, x)<\delta\})$ and for some $n>0$ and $y \in B_{\varepsilon^{*}}(x) \cap A$, we have $T^{n}(y) \in$ $B_{\varepsilon^{*}}(x)$ and $a(n, y) \geq 0$. Moreover, if for some $n_{x}>0, T^{n_{x}}(x) \in B_{\varepsilon^{*}}(x)$ and $a\left(n_{x}, x\right)<0$, then we can find $n>0$ and $y \in B_{\varepsilon^{*}}(x) \cap A$ as above such that $a(n, y)>0$.

Proof. First, note that no point in $A$ is periodic, as we are supposing that no $g$-maximizing measures for $T$ are periodic. So, given $x \in A$, as it is not fixed, there exists $0<\varepsilon^{*}<\varepsilon / 10$ such that $T\left(B_{\varepsilon^{*}}(x)\right) \cap B_{\varepsilon^{*}}(x)=\emptyset$.

Since $x$ is $T$-recurrent, there exists $N>0$ such that $T^{N}(x) \in B_{\varepsilon^{*}}(x)$. Let

$$
C=\sum_{i=0}^{N-1} g_{0} \circ T^{i}(x)
$$

Now we analyze two possibilities. 
- $C<0$. Since $T^{N}(x) \in B_{\varepsilon^{*}}(x)$, there exists $0<\bar{\delta}_{1}<\varepsilon^{*}$ such that for all $w \in B_{\bar{\delta}_{1}}(x)$,

$$
T^{N}(w) \in B_{\varepsilon^{*}}(x) \text { and } \sum_{i=0}^{N-1} g_{0} \circ T^{i}(w)<C / 2<0 .
$$

Now let $\delta_{1}=\min \left\{\bar{\delta}_{1} / 10,-C / 10\right\}$. As $x \in \operatorname{supp}(\mu)$ and $\mu(A)=1$, we see that $\mu\left(B_{\delta_{1}}(x) \cap A\right)>0$. So Lemma 1 implies that there exist $z^{*} \in B_{\delta_{1}}(x) \cap A$ and $N^{*}$ with $\left|N^{*}\right|>N$ such that

$$
T^{N^{*}}\left(z^{*}\right) \in B_{\delta_{1}}(x) \text { and }\left|a\left(N^{*}, z^{*}\right)\right|<\delta_{1} .
$$

If $N^{*}>0$, then let $z=z^{*}$; otherwise, let $z=T^{N^{*}}\left(z^{*}\right) \in A$. In both cases, let $N^{\prime}=\left|N^{*}\right|$. Thus, (4) and (5) imply that

$$
C / 10<a\left(N^{\prime}, z\right)=a(N, z)+a\left(N^{\prime}-N, T^{N}(z)\right)<C / 2+a\left(N^{\prime}-N, T^{N}(z)\right)
$$

and so

$$
a\left(N^{\prime}-N, T^{N}(z)\right)>-2 C / 5>0 .
$$

From the choice of $z$ and $\delta_{1}$, we infer that $y=T^{N}(z) \in B_{\varepsilon^{*}}(x) \cap A$, $T^{N^{\prime}-N}(y) \in B_{\delta_{1}}(x) \subset B_{\varepsilon^{*} / 10}(x)$, and this proves the lemma with $n=N^{\prime}-N$.

- $C \geq 0$. The lemma is proved by setting $n=N$ and $y=x$.

Now, for a given $\varepsilon>0$ and some fixed $x_{0} \in A$, Lemma 2 implies that there are two possibilities:

1. There exist $n>0$ and $0<\varepsilon^{*}<\varepsilon / 10$ such that $T\left(B_{\varepsilon^{*}}\left(x_{0}\right)\right) \cap B_{\varepsilon^{*}}\left(x_{0}\right)$ $=\emptyset, T^{n}\left(x_{0}\right) \in B_{\varepsilon^{*}}\left(x_{0}\right),\left\{T\left(x_{0}\right), \ldots, T^{n-1}\left(x_{0}\right)\right\} \cap B_{\varepsilon^{*}}\left(x_{0}\right)=\emptyset, a\left(n, x_{0}\right)$ $=0$ and for all $y \in B_{\varepsilon^{*}}\left(x_{0}\right)$ and $i>0$ such that $T^{i}(y) \in B_{\varepsilon^{*}}\left(x_{0}\right)$, we have $a(i, y) \leq 0$.

2. There exists $0<\varepsilon^{*}<\varepsilon / 10$ such that $T\left(B_{\varepsilon^{*}}\left(x_{0}\right)\right) \cap B_{\varepsilon^{*}}\left(x_{0}\right)=\emptyset$ and for some $\widetilde{x} \in B_{\varepsilon^{*}}\left(x_{0}\right)$ and $n>0, T^{n}(\widetilde{x}) \in B_{\varepsilon^{*}}\left(x_{0}\right)$ and $a(n, \widetilde{x})>0$. We then set

$$
K:=a(n, \widetilde{x}) / n>0 .
$$

If the first possibility occurs, we proceed as follows:

Take a simple arc $\gamma \subset B_{\varepsilon^{*}}\left(x_{0}\right)$ with endpoints $x_{0}$ and $T^{n}\left(x_{0}\right)$. Let $V \subset B_{\varepsilon^{*}}\left(x_{0}\right)$ be a sufficiently small open neighborhood of $\gamma$. Construct a homeomorphism $\widetilde{h}$ of $X$, which is the identity outside $V$ and maps $T^{n}\left(x_{0}\right)$ to $x_{0}$. Define a new homeomorphism by $\widetilde{T}=\widetilde{h} \circ T$. Clearly, $\widetilde{T}$ is $\varepsilon^{*}-\mathcal{C}^{0}$-close to $T$ (note that we have just presented a proof of the $\mathcal{C}^{0}$-closing lemma: we perturbed $T$ inside $B_{\varepsilon^{*}}\left(x_{0}\right)$ in order to close the partial orbit of $\left.x_{0}\right)$, and for the perturbed mapping $\widetilde{T}$, we have

$$
Q_{\widetilde{T}}\left(g_{0}\right)=\sup _{\mu \in M_{\text {inv }}(\widetilde{T})} \int_{X} g_{0} d \mu=\frac{1}{n} a\left(n, x_{0}\right)=0, \quad \text { so } \quad Q_{\widetilde{T}}(g)=Q_{T}(g),
$$


and the main theorem is proved. This is because every time a point in $B_{\varepsilon^{*}}\left(x_{0}\right)$ returns to $B_{\varepsilon^{*}}\left(x_{0}\right)$, it has a partial average less than or equal to zero. Thus the perturbation we apply to $T$ which is supported in $B_{\varepsilon^{*}}\left(x_{0}\right)$ and closes the orbit of $x_{0}$ cannot increase partial averages of any other point when it returns to $B_{\varepsilon^{*}}\left(x_{0}\right)$. After this perturbation, we may have created an invariant measure $\mu^{*}$ for $\widetilde{T}$ which is not periodic, but $\int_{X} g_{0} d \mu^{*} \leq 0$. With the type of perturbation used, it is not possible to create a $\widetilde{T}$-invariant measure with average greater than zero.

In the rest of the paper we will deal with the second possibility.

The proof consists of two parts:

- Part 1: here we make use of the point $\widetilde{x}$ and some compactness arguments to perturb $T$ in order to create a periodic orbit $O_{P}$ with positive $g_{0}$ average for a nearby mapping $T_{1}$, satisfying many technical assumptions. We point out that $T_{1}$ may have other invariant measures with $g_{0}$ average larger than the $g_{0}$ average on $O_{P}$, and this is the most difficult hurdle to overcome in our result, which we do in part 2 . The support of this periodic orbit includes a point $P$ close to $\widetilde{x}$, and $T_{1}$ only differs from $T$ in a small open set containing $P, V_{\eta}(\alpha)$, yet to be defined. The technical assumptions ensure that there is an even smaller neighborhood of $P, C_{\delta}(P) \cap V_{\eta}(\alpha)$, such that trajectories with a $g_{0}$ average for $T_{1}$ greater than the $g_{0}$ average of $P$ must visit this smaller neighborhood frequently.

- Part 2: the periodic orbit created above for $T_{1}$ is transformed, by another perturbation which fixes it, into a topological source for the new mapping $T_{2}$, that is, it becomes a repeller. This is used to show that after the two perturbations $\left(T \rightarrow T_{1}\right.$ and $\left.T_{1} \rightarrow T_{2}\right)$, we do not create any measure with average larger than the average of the periodic orbit created in part 1 . This is because the repelling nature of the periodic orbit does not let points follow it for a long time. Every nonperiodic orbit of $T_{2}$ must eventually have the property that consecutive returns to the set $V_{\eta}(\alpha)$ cannot both lie in $C_{\delta}(P)$, thus "losing average" on at least one of the orbit segments.

Going back to the proof itself, if $\varepsilon^{*}>0$ in the second possibility is sufficiently small, then $B_{\varepsilon^{*}}\left(x_{0}\right)$ is contained in some local chart of $X$. Since in the rest of the paper we will modify $T$ only inside $B_{\varepsilon^{*}}\left(x_{0}\right)$, we will treat, without loss of generality, $B_{\varepsilon^{*}}\left(x_{0}\right)$ as a subset of $\mathbb{R}^{n}$, and we will make use of its geometrical and convexity properties.

First, note that

$$
\limsup _{l \rightarrow \infty}\left(\sup _{y \in X} \frac{1}{l} a(l, y)\right)=0
$$


so there exists $N>n>0$ such that for all $l \geq N$ and $y \in X$,

$$
\frac{1}{l} a(l, y)<K / 2 .
$$

Now we will prove that among all pairs of points $z, T^{i}(z)(i>0)$ belonging to the straight line segment $\overline{\widetilde{x} T^{n}(\widetilde{x})}$, we can pick one with largest possible partial average and shortest distance. First, note that from (6) we just have to consider the first $N$ iterates of the straight line segment $\widetilde{\widetilde{x} T^{n}(\widetilde{x})}$. To be more precise, for $i \in\{1, \ldots, N\}$ consider the sets

$$
\operatorname{Ret}_{i}=\left\{z \in \overline{\widetilde{x} T^{n}(\widetilde{x})}: T^{i}(z) \in \overline{\widetilde{x} T^{n}(\widetilde{x})}\right\}
$$

and the associated partial averages

$$
\operatorname{Av}_{i}(z)=\frac{a(i, z)}{i}=\frac{1}{i} \sum_{l=0}^{i-1} g_{0} \circ T^{l}(z) .
$$

One can pick $P, T^{m}(P) \in \overline{\widetilde{x} T^{n}(\widetilde{x})}$, for some $0<m<N$, such that $\operatorname{Av}_{m}(P)=$ $\max _{1 \leq i \leq N}\left\{\left.\max \operatorname{Av}_{i}\right|_{\operatorname{Ret}_{i}}\right\}$ and among all pairs $z, T^{i}(z) \in \overline{\widetilde{x} T^{n}(\widetilde{x})}$ with $\operatorname{Av}_{i}(z)$ $=\operatorname{Av}_{m}(P), d\left(P, T^{m}(P)\right)$ has the smallest value. If more than one pair satisfies the above, any of them will fit (clearly it is possible that $P=\widetilde{x}$ and $m=n<N)$.

Below we give four properties of the pair $P, T^{m}(P)$ that will be used in the rest of the paper.

1. For all $z \in \overline{\widetilde{x} T^{n}(\widetilde{x})}$ and $i>0$ such that $T^{i}(z) \in \overline{\widetilde{x} T^{n}(\widetilde{x})}$, we have

$$
\frac{1}{i} a(i, z) \leq \frac{1}{m} a(m, P)=\psi(P) \geq K,
$$

where for now, $\psi(P)$ is just a symbol for the partial average at $P$. A complete definition of the function $\psi$ is given after formula (10). Clearly this definition will agree with the above one at $P$.

2. For all $z \in \overline{P T^{m}(P)}, z \neq P$, and $i>0$ such that $T^{i}(z) \in \overline{P T^{m}(P)}$,

$$
\frac{1}{i} a(i, z)<\psi(P)
$$

because from the choice of $P$, if $a(i, z) / i=\psi(P)$, then the only possibility would be $z=T^{m}(P)$ and $T^{i}(z)=P$, which means that the orbit of $P$ is periodic with positive average, a contradiction.

3. For $i=1, \ldots, m-1, T^{i}(P) \notin \overline{P T^{m}(P)}$. To see this, note that if for some $1 \leq i \leq m-1, T^{i}(P) \in \overline{P T^{m}(P)}$, then as at least one of the numbers

$$
\frac{1}{i} a(i, P), \quad \frac{1}{m-i} a\left(m-i, T^{i}(P)\right)
$$

is greater than or equal to $\psi(P)$, we could change $P$ and $m$ to get a smaller straight line segment. 
4. If $l>m$ and $T^{l}(P) \in \overline{P T^{m}(P)}$, then

$$
\frac{1}{l} a(l, P)<\psi(P)-c_{1}
$$

for some constant $c_{1}>0$. As we already said, condition (6) implies that partial averages taken for sufficiently large times are small, so we just have to consider $m<l<N$. Thus inequality (7) follows as property 2 above.

From now on, denote by $\alpha$ the segment $\overline{P T^{m}(P)}$ and by $C_{\delta}(P)$ a closed cube of side $2 \delta>0$ centered at $P$. If we fix a sufficiently small $\delta>0$, then:

- $d\left(T^{m}\left(C_{\delta}(P)\right), C_{\delta}(P)\right)>d\left(P, T^{m}(P)\right) / 2>10 \delta$, which, in particular, implies that $T^{m}\left(C_{\delta}(P)\right) \cap C_{\delta}(P)=\emptyset$.

- For all $0<i<N$, where $N$ is defined in (6), we have: if $T^{i}(P) \notin \alpha$, then $T^{i}\left(C_{\delta}(P)\right) \cap\left(\alpha \cup C_{\delta}(P)\right)=\emptyset$, and if $T^{i}(P) \in \alpha$ and $i \neq m(i>m)$, then for the $c_{1}>0$ defined in (7), we have

$$
\frac{1}{i} a(i, z)<\psi(P)-c_{1} \quad \text { for all } z \in C_{\delta}(P) .
$$

Associated to the $\delta>0$ chosen above, there exists a $0<c_{2}<c_{1}$ such that if $x, T^{i}(x) \in \alpha$ for some integer $i>0$ and

$$
\frac{1}{i} a(i, x) \geq \psi(P)-c_{2},
$$

then $i=m$ and $x \in C_{\delta / 2}(P)$. We note that, if $c_{2}$ did not exist, then property 2 above would be contradicted.

In the following lemma, remember that $\alpha$ is the straight line segment $\overline{P T^{m}(P)}$, and $\delta>0$ and $c_{2}>0$ have been chosen above.

LEMma 3. There exists an $\eta \in(0, \delta)$ such that if both $z$ and $T^{l}(z)$ belong to $V_{\eta}(\alpha) \subset B_{\varepsilon^{*}}\left(x_{0}\right)$ (the $\eta$-neighborhood of $\alpha$ ) for some $l>0$, and $a(l, z) / l \geq$ $\psi(P)-c_{2}$, then $z \in C_{\delta}(P) \cap V_{\eta}(\alpha)$ and $l=m$. Furthermore, $a(m, z) / m<$ $\psi(P)+c_{2} / 2 m$.

Proof. Suppose that for each integer $k>0$, there exists a point $z_{k} \in$ $V_{1 / k}(\alpha) \backslash C_{\delta}(P)$ such that for some $0<n_{k}<N$ (see (6) for the definition of $N), T^{n_{k}}\left(z_{k}\right) \in V_{1 / k}(\alpha)$ and $a\left(n_{k}, z_{k}\right) / n_{k} \geq \psi(P)-c_{2}$. Taking subsequences if necessary, this implies that there exists a point $z^{*} \in \alpha \backslash C_{2 \delta / 3}(P)$ such that (for some $\left.n^{*}>0\right) T^{n^{*}}\left(z^{*}\right) \in \alpha$ and $a\left(n^{*}, z^{*}\right) / n^{*} \geq \psi(P)-c_{2}$, contradicting the choice of $\delta>0$ and $c_{2}>0$ (see (9)).

So if $k>0$ is large enough, then for every $z \in V_{1 / k}(\alpha) \backslash C_{\delta}(P)$ such that for some $0<l<N, T^{l}(z) \in V_{1 / k}(\alpha)$, we have $a(l, z) / l<\psi(P)-c_{2}$.

Since $C_{\delta}(P)$ is closed, if $k>0$ is sufficiently large, then for every $0<$ $i<N$ such that $T^{i}(P) \notin \alpha$, we have

$$
T^{i}\left(C_{\delta}(P)\right) \cap\left(V_{1 / k}(\alpha) \cup C_{\delta}(P)\right)=\emptyset
$$


(this follows from the choice of $\delta>0$ ). Now suppose $z \in C_{\delta}(P) \cap V_{1 / k}(\alpha)$ and $T^{l}(z) \in V_{1 / k}(\alpha)$ for some $0<l<N$. From the previous remark, if $k>0$ is large enough, then $T^{l}(P) \in \alpha$. In this case, if $l \neq m$, from (8) we see that $a(l, z) / l<\psi(P)-c_{1}<\psi(P)-c_{2}$ for all $z \in C_{\delta}(P)$.

Finally, suppose that for each integer $k>0$, there exists a $y_{k} \in V_{1 / k}(\alpha)$ such that $T^{m}\left(y_{k}\right) \in V_{1 / k}(\alpha)$ and $a\left(m, y_{k}\right) / m \geq \psi(P)+c_{2} / 2 m$. This would imply the existence of a point $\bar{y} \in \alpha$ such that $T^{m}(\bar{y}) \in \alpha$ and $a(m, \bar{y}) / m>$ $\psi(P)$. But this contradicts the choice of $P$.

So the lemma is proved.

The following fact will be useful in the proof of the main theorem:

FACT 1. If $\bar{T} \in \operatorname{Hom}(X)$ and $\nu$ is a $\bar{T}$-invariant Borel probability measure such that $\int_{X} g_{0} d \nu>0$, then by Birkhoff's ergodic theorem, there is an $x \in X$ such that $\lim _{n \rightarrow \infty} n^{-1} \sum_{i=0}^{n-1} g_{0} \circ \bar{T}^{i}(x)>0$.

Now let $\eta^{\prime} \in(0, \eta)$, where $\eta$ comes from Lemma 3 , be sufficiently small such that $T^{i}(P) \notin V_{\eta^{\prime}}(\alpha)$ for $i=1, \ldots, m-1$. We define $E:=V_{\eta^{\prime}}(\alpha)$.

As $E$ is a convex set, we can define, for every point $x$ in $E$, the Minkowski function $M$ with respect to $P$ as

$$
M: E \rightarrow[0,1], \quad M(x)=\inf \left\{t \in[0,1]: P+\frac{1}{t}(x-P) \in E\right\} .
$$

The following properties follow almost immediately from the definition:

- $M$ is continuous and monotone on radial lines;

- $M(x)=0 \Leftrightarrow x=P$, and $M(x)=1 \Leftrightarrow x \in \partial E$.

Let $a_{0}=M\left(T^{m}(P)\right)<1$ and set

$$
E_{L}=M^{-1}\left(\left[0,\left(a_{0}+1\right) / 2\right]\right), \quad E_{S}=M^{-1}\left(\left[0,\left(2 a_{0}+1\right) / 3\right]\right) .
$$

As $1>\left(a_{0}+1\right) / 2>\left(2 a_{0}+1\right) / 3$, we have

$$
\overline{P T^{m}(P)}=\alpha \subset E_{S} \subset E_{L} \subset E,
$$

with all inclusions proper (see Figure 1).

Now, since $E_{S}$ is homeomorphic to the closed ball in $\mathbb{R}^{n}$, there exists a homeomorphism $h_{S}$ of $\mathbb{R}^{n}$ such that $h_{S}$ is the identity outside $E_{S}$, and $h_{S}\left(T^{m}(P)\right)=P$.

Consider now the homeomorphism $T_{1}=h_{S} \circ T$. Clearly, $T_{1}=T$ outside $T^{-1}\left(E_{S}\right)$.

Note that $P$ is a periodic point for $T_{1}$ and if we consider the counting measure

$$
\nu=\frac{1}{m} \sum_{i=0}^{m-1} \delta_{T_{1}^{i}(P)}
$$




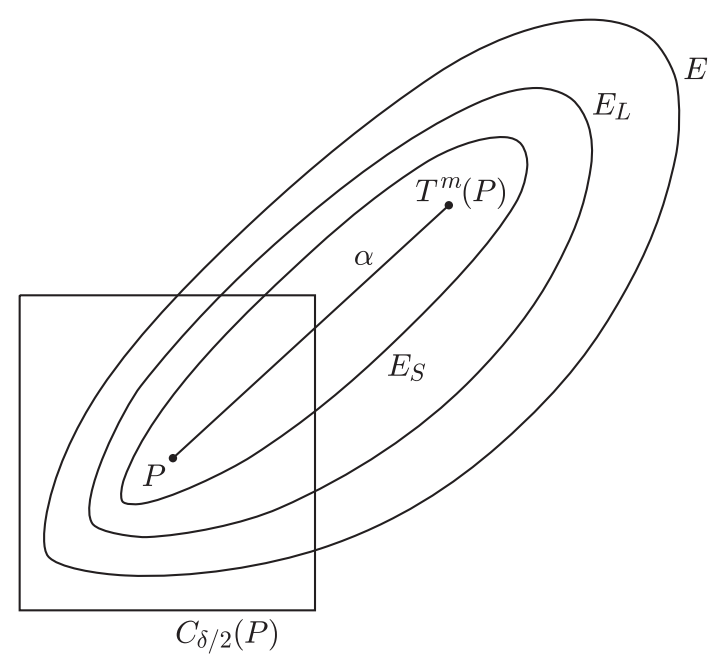

Fig. 1. Diagram showing the sets $\alpha, E_{S}, E_{L}$ and $E$

on the orbit of $P$, then $\nu$ is $T_{1}$-invariant and $\int_{X} g_{0} d \nu=\psi(P)>0$. Also, if a point $x$ does not visit $E_{S}$ infinitely many times, then clearly, since $T_{1}$ only differs from $T$ inside $E_{S}, \lim _{n \rightarrow \infty} n^{-1} \sum_{i=0}^{n-1} g_{0} \circ T_{1}^{i}(x) \leq 0$. This still does not prove the desired result, because a trajectory recurrently visiting $E_{S}$ could, in principle, have a greater $g_{0}$ average than $\psi(P)$, especially given that $P$ may fail to be a maximum for $m^{-1} \sum_{i=0}^{m-1} g_{0} \circ T^{i}(x)$ with $x \in E_{S}$ very close to $P$.

Our proof comes from the construction of another homeomorphism $T_{2}=$ $h \circ T_{1}$, leaving the orbit of $P$ unaltered $(h(P)=P)$. The homeomorphism $h$ differs from the identity only in $E$ and so $T_{2}$ is $\varepsilon$-close to $T$ in the space $\operatorname{Hom}(X)$. For $x \in E(x \neq P)$, the mapping $h$ will be of the form

$$
h(x)=P+\frac{f(M(x))}{M(x)}(x-P), \quad f:[0,1] \rightarrow[0,1],
$$

where $f$ is continuous $(f(0)=0$ and $f(1)=1)$, strictly increasing and $f(r)>r$ for $0<r<1$. In order to see that $h$ is injective on each straight line segment $r$ starting at $P$ and ending at a point $e(r) \in \partial E$, and leaves it invariant, fixing its endpoints, note that for any $x \in r$,

$$
h(x)=P+f(M(x)) \frac{x-P}{M(x)}=P+f(M(x))[e(r)-P] .
$$

To understand why $h: E \rightarrow E$ is a homeomorphism, it remains to show that $h$ is continuous at $P$, and this follows from

$$
\|h(x)-P\|=f(M(x))\left\|\frac{x-P}{M(x)}\right\| \leq f(M(x))\left[\max _{e \in \partial E} d(P, e)\right] \stackrel{x \rightarrow P}{\longrightarrow} 0 .
$$


As $f(M(x)) \leq 1$, we deduce that if $x \in E$ then $h(x) \in E$. And for points $x \in E$, as

$$
\frac{1}{f(M(x))}(h(x)-P)=\frac{1}{M(x)}(x-P),
$$

we obtain

$$
M(h(x))=f(M(x)) .
$$

In order to finish the construction of $h$, first we consider the set $E_{r}=$ $E \cap \bigcup_{i \in \mathbb{N}^{*}} T_{1}^{-i}(E)$ of points in $E$ eventually returning to $E$. In $E_{r}$ we define two functions,

$$
N_{\text {ret }}(x)=\inf \left\{j \in \mathbb{N}^{*} \mid T_{1}^{j}(x) \in E\right\}=\inf \left\{j \in \mathbb{N}^{*} \mid T^{j}(x) \in E\right\},
$$

which is the first return time to the set $E$ for $T_{1}, T$ and $T_{2}$, and

$$
\psi(x)=\frac{1}{N_{\text {ret }}(x)} \sum_{i=0}^{N_{\text {ret }}(x)-1} g_{0} \circ T_{1}^{i}(x)=\frac{1}{N_{\text {ret }}(x)} a\left(N_{\text {ret }}(x), x\right),
$$

the average of $g_{0}$ on the orbit of $x$ until the next return.

Clearly $\left.T_{1}\right|_{X \backslash E}=\left.T\right|_{X \backslash E}$, and moreover, the two definitions for $\psi(P)$ agree.

Note that for any $x$ in $E_{r}$, it is also true that $T_{2}^{N_{\text {ret }}(x)}(x) \in E$, and $N_{\text {ret }}(x)$ is the smallest strictly positive integer for which this holds.

The choice of $\delta$ (see the proof of Lemma 3 and definition of $C_{\delta}(P)$ before this lemma) ensure that $T^{i}\left(C_{\delta}(P)\right) \cap\left[E \cup C_{\delta}(P)\right]=\emptyset$ for $1 \leq i \leq m-1$, so for any $x \in C_{\delta}(P) \cap E_{r}$ we have $N_{\text {ret }}(x) \geq m$. Since $P \in C_{\delta}(P)$, and taking into consideration that $T_{1}^{m}(P)=P$ is an interior point of $E$, there is a $\delta_{2}>0$ such that for any $x$ satisfying $d(x, P) \leq \delta_{2}$, we have $x \in E_{L} \cap C_{\delta}(P)$ and $T_{1}^{m}(x) \in E$. Therefore, $N_{\text {ret }}(x)=m$ for all $x$ in $B_{\delta_{2}}(P)$.

We will choose $f$ so that $T_{2}^{m}\left(B_{\delta_{2}}(P)\right) \supset E_{L}$. We will also use $f$ to ensure that for a chained sequence of neighborhoods of $P$ whose intersection is $\{P\}$, $T_{2}^{m}$ always takes a "smaller" neighborhood onto a larger one, so that $P$ is a topological source for $T_{2}^{m}$. So, consider the sequence $\left(b_{n}\right)_{n \in \mathbb{N}}$ of real numbers defined in the following manner:

Let $\widetilde{b}$ be any real such that $M^{-1}([0, \widetilde{b}])$ is contained in $B_{\delta_{2}}(P)$ and let

$$
b_{k}=\min \left\{2^{-k} \widetilde{b}, \inf _{x \in \partial\left(M^{-1}\left(\left[0,2^{-k} \widetilde{b}\right]\right)\right)}\left\{M\left(T_{1}^{m}(x)\right)\right\}\right\}, \quad k \geq 1,
$$

so that $T_{1}^{m}\left(M^{-1}\left(\left[0,2^{-k} \widetilde{b}\right]\right)\right) \supset M^{-1}\left(\left[0, b_{k}\right]\right)$, and $T_{1}^{m}\left(B_{\delta_{2}}(P)\right) \supset M^{-1}\left(\left[0, b_{1}\right]\right)$.

Now, if we take $f$ to be an affine function on $\left[b_{1}, 1\right]$ and on each of the intervals $\left[b_{k+1}, b_{k}\right]$ (note that $\left(b_{k}\right)$ is strictly decreasing and tends to 0 ), and such that

$$
f(1)=1, \quad f\left(b_{1}\right)=\frac{a_{0}+1}{2}, \quad f\left(b_{k+1}\right)=2^{-k} \widetilde{b}, \quad f(0)=0,
$$


then, using (10), we obtain

$$
h\left(M^{-1}\left(\left[0, b_{1}\right]\right)\right)=E_{L}, \quad h\left(M^{-1}\left(\left[0, b_{k+1}\right]\right)\right)=M^{-1}\left(\left[0,2^{-k} \widetilde{b}\right]\right),
$$

and

$$
\begin{gathered}
T_{2}^{-m}\left(E_{L}\right) \subset M^{-1}([0, \widetilde{b}]) \subset B_{\delta_{2}}(P) \subset E_{L}, \\
T_{2}^{-m}\left(M^{-1}\left(\left[0,2^{-k} \tilde{b}\right]\right) \subset M^{-1}\left(\left[0,2^{-(k+1)} \tilde{b}\right]\right) .\right.
\end{gathered}
$$

This construction turns the $T_{2}$-orbit of $P$ into a source. Also, for all $l \geq 0$,

$$
T_{2}^{-(l+1) m}\left(E_{L}\right) \subset T_{2}^{-l m}\left(E_{L}\right) \text { and } \bigcap_{l=0}^{\infty} T_{2}^{-l m}\left(E_{L}\right)=\{P\} .
$$

Thus, for any $x \in E_{L}, x \neq P$, there is a final time $n_{f}(x)$ such that if $i>n_{f}(x)$, then $T_{2}^{i}(x) \notin E_{L}$.

Finally, let us consider the following simple lemma:

Lemma 4. If $x \notin C_{\delta}(P)$, then $h(x) \notin C_{\delta}(P)$.

Proof. If $x \notin \operatorname{int}(E)$ or $x=P$, then $h(x)=x$. Now, if $x \in \operatorname{int}(E), x \neq P$, then since $1>f(M(x))>M(x)>0$, the point

$$
x=\left(\frac{(M(x))^{2}}{M(x) f(M(x))}\right) h(x)+\left(\frac{(f(M(x))-M(x)) M(x)}{M(x) f(M(x))}\right) P
$$

always belongs to the segment $\overline{P h(x)}$. But $C_{\delta}(P)$ is convex and so the result follows.

The main result will follow from Fact 1 and

LEMma 5. For any $x \in X$,

$$
\limsup _{n \rightarrow \infty} \frac{1}{n} \sum_{i=0}^{n-1} g_{0} \circ T_{2}^{i}(x) \leq \psi(P) .
$$

Proof. Clearly, we can assume that $x$ returns infinitely many times to $E$, and that $x$ belongs to $E \backslash E_{L}$. This excludes the possibility that $x$ or any of its forward iterates lies in $E_{S} \subset E_{L}$.

Now, if $x$ returns infinitely many times to $E$, we can consider the sequence of return times $\left(n_{i}\right)_{i \in \mathbb{N}}$ given by

$$
n_{0}=0, \quad n_{i+1}=n_{i}+N_{\text {ret }}\left(T_{2}^{n_{i}}(x)\right),
$$

and the associated sequence of returns

$$
x_{0}=x, \quad x_{i}=T_{2}^{n_{i}}(x) .
$$

Let $n_{\left(m_{j}\right)}$ be the subsequence (possibly infinite) of return times where $x_{\left(m_{j}\right)}$ belongs to $C_{\delta}(P)$, and such that $N_{\text {ret }}\left(x_{\left(m_{j}\right)}\right)=m$. 
In these situations, since $x_{\left(m_{j}\right)} \notin E_{S}$, we have $T^{m}\left(x_{\left(m_{j}\right)}\right)=T_{1}^{m}\left(x_{\left(m_{j}\right)}\right)$ and

$$
x_{\left(m_{j}\right)+1}=h \circ T^{m}\left(x_{\left(m_{j}\right)}\right),
$$

and since $T^{m}\left(x_{\left(m_{j}\right)}\right) \notin C_{\delta}(P)$, from Lemma 4 the same is true for $x_{\left(m_{j}\right)+1}$. This implies that $m_{j+1}>m_{j}+1$, and so, if a return belongs to $C_{\delta}(P)$, the subsequent one does not. We will assume, with no loss in generality, that $m_{0}=0$.

Now, from Lemma 3, if $\psi\left(x_{i}\right)>\psi(P)-c_{2}$, then $x_{i} \in C_{\delta}(P)$ and $i=m_{j}$ for some $j$. Also for any positive integer $j, \psi\left(x_{j}\right)<\psi(P)+c_{2} / 2 m$. So

$$
\begin{aligned}
\sum_{i=0}^{n_{\left(m_{j}\right)}-1} g_{0} \circ T_{2}^{i}(x)= & \sum_{i=0}^{m_{j}-1} N_{\text {ret }}\left(x_{i}\right) \psi\left(x_{i}\right) \\
= & \sum_{l=0}^{j-1}\left[m \psi\left(x_{\left(m_{l}\right)}\right)+N_{\text {ret }}\left(x_{\left(m_{l}\right)+1}\right) \psi\left(x_{\left(m_{l}\right)+1}\right)\right. \\
& \left.+\sum_{i=m_{l}+2}^{m_{l+1}-1} N_{\text {ret }}\left(x_{i}\right) \psi\left(x_{i}\right)\right],
\end{aligned}
$$

but since all returns $x^{\prime}$ between $x_{\left(m_{l}\right)}$ and $x_{\left(m_{l+1}\right)}$ lie outside $C_{\delta}(P)$ or, if they lie in $C_{\delta}(P), N_{\text {ret }}\left(x^{\prime}\right) \neq m$, by the consequence of Lemma 3 stated just before (11), we get

$$
\begin{aligned}
m \psi\left(x_{\left(m_{l}\right)}\right)+ & N_{\text {ret }}\left(x_{\left(m_{l}\right)+1}\right) \psi\left(x_{\left(m_{l}\right)+1}\right) \\
& <m\left(\psi(P)+c_{2} / 2 m\right)+N_{\text {ret }}\left(x_{\left(m_{l}\right)+1}\right)\left(\psi(P)-c_{2}\right) \\
& <\left(m+N_{\text {ret }}\left(x_{\left(m_{l}\right)+1}\right)\right) \psi(P)=\left[\left(n_{\left(m_{l}\right)+2}\right)-\left(n_{\left(m_{l}\right)}\right)\right] \psi(P),
\end{aligned}
$$

showing that the "loss of average" between returns in the portion of the orbit starting outside $C_{\delta}(P)$ is larger than the possible gain in the portion starting at $C_{\delta}(P)$. Also, if $i \in\left[m_{l}+2, m_{l+1}-1\right]$, then again $\psi\left(x_{i}\right)<\psi(P)-c_{2}<\psi(P)$. So

$$
\sum_{i=m_{l}+2}^{m_{l+1}-1} N_{\text {ret }}\left(x_{i}\right) \psi\left(x_{i}\right)<\left(n_{\left(m_{l+1}\right)}-n_{\left(m_{l}\right)+2}\right)\left(\psi(P)-c_{2}\right) .
$$

Together, the two inequalities above give (remember (11))

$$
\sum_{i=0}^{n_{\left(m_{j}\right)}-1} g_{0} \circ T_{2}^{i}(x)<n_{\left(m_{j}\right)} \psi(P) .
$$

If $m_{j}$ is an infinite sequence, then

$$
\limsup _{j \rightarrow \infty} \frac{1}{n_{\left(m_{j}\right)}} \sum_{i=0}^{n_{\left(m_{j}\right)}-1} g_{0} \circ T_{2}^{i}(x) \leq \psi(P),
$$


and if $m_{j}$ is a finite sequence, then clearly

$$
\limsup _{i \rightarrow \infty} \frac{1}{n_{i}} \sum_{l=0}^{n_{i}-1} g_{0} \circ T_{2}^{l}(x) \leq \psi(P)-c_{2},
$$

ending the proof of the lemma and also of the main theorem.

\section{References}

[1] J. Aaronson, An Introduction to Infinite Ergodic Theory, Math. Surveys Monogr. 50, Amer. Math. Soc., Providence, RI, 1997.

[2] G. Atkinson, Recurrence of cocycles and random walks, J. London Math. Soc. (2) 13 (1976), 486-488.

[3] T. Bousch, Le poisson n'a pas d'arêtes, Ann. Inst. H. Poincaré Probab. Statist. 36 (2000), 489-508.

[4] O. Jenkinson, Every ergodic measure is uniquely maximizing, Discrete Contin. Dynam. Systems 16 (2006), 383-392.

[5] —, Ergodic optimization, ibid. 15 (2006), 197-224.

[6] R. Mañé, An ergodic closing lemma, Ann. of Math. 116 (1982), 503-540.

[7] J. Mather, Action minimizing invariant measures for positive definite Lagrangian systems, Math. Z. 207 (1991), 169-207.

[8] K. Schmidt, Lectures on Cocycles of Ergodic Transformation Groups, Math. Inst., Univ. of Warwick, 1976.

Instituto de Matemática e Estatística

Universidade de São Paulo

Rua do Matão 1010, Cidade Universitária

05508-090 São Paulo, SP, Brazil

E-mail: fabiotal@ime.usp.br

sazanata@ime.usp.br

Received 30 November 2006;

in revised form 3 April 2008 